\title{
EMBO workshop on cytotoxicity, cell death and the immune system
}

\author{
A Anel ${ }^{*, 1}$, C Bleackley ${ }^{2}$, C Borner ${ }^{3}$, P Golstein ${ }^{4}$, PH Krammer ${ }^{5}$, A Müllbacher ${ }^{6}$, J Pardo $^{1,7}$, MM Simon $^{8}$ and JA Trapani ${ }^{9}$ \\ Cell Death and Differentiation (2009) 16, 790-793. doi:10.1038/cdd.2009.4
}

Zaragoza, Spain, 17-20 September 2008

120 researchers from 24 different countries participated in this workshop, and the questions and debates, especially at the two poster sessions, were really vivid and enriching. The workshop covered different areas of cell death research, not all exclusively related with the immune system. The different scientific subjects treated during the eight Symposia and three plenary lectures, and summarized below, constitute an abbreviated update of the state of the art in the different thematics.

The first plenary by Pierre Golstein (Marseille) and Peter Krammer (Heidelberg) was an authorized 'Overview of cell death'. Pierre Golstein made a historical and evolutionary introduction on cell death research, and gave molecular keys to define the three different types of programmed cell death: apoptotic, autophagic and necrotic. He also showed how the two latter forms of cell death could be studied in the Dictyostelium discoideum model. Peter Krammer discussed new data on activation-induced cell death (AICD) of activated T cells (ATC), which implicates TCR-mediated activation of PKC $\theta$ and mitochondrial complex I-mediated generation of $\mathrm{H}_{2} \mathrm{O}_{2}$ in the activation of NF- $\kappa$ B and AP-1. This leads to transcriptional expression of the CD95 ligand (CD95L), which binds to the death receptor CD95 and induces 'fratricidal' or 'autocrine' T cell death.

\section{Symposium A. Modes of Cell Death}

Among the three main types of cell death currently believed to take place in animal cells, apoptosis, which is caspasedependent, has been intensively studied. The caspaseindependent non-apoptotic cell death types, autophagic and necrotic, have been far less well studied. It is becoming clear that these non-apoptotic cell death types can be quite important especially in pathological contexts (infectious, ischemic and degenerative). Peter Vandenabeele (Ghent) compared necrotic cell death induced by TNF or by $\mathrm{H}_{2} \mathrm{O}_{2}$ using time-lapse microscopy. The former led to the complex I-dependent generation of reactive oxygen species and involves the RIP1 kinase, whereas the latter could be inhibited by iron chelators blocking Fenton reactions leading to lysosomal membrane permeabilization. At least TNF-mediated necrosis was regulated, contributing to the notion that necrotic cell death can be programmed. Nele Vanlangenakker, from the same laboratory, reported on an RNAi screen of the mouse kinome. A number of promising candidates for regulating necrotic cell death have been identified in this manner, and are being validated. Francis Chan (Worcester) similarly screened a small RNAi library to identify kinases involved in TNF-induced programmed necrosis. This screen revealed several RIP family kinases, mitochondrial-associated and MAP kinases, which are under further investigation. Adi Kimchi (Rehovot), using systems level analysis, showed that autophagic cell death involved among others, the DAP kinase and a new isoform of p19ARF, called smARF. Beth Levine (Dallas) described two novel functions of the autophagy pathway, in promoting the clearance of apoptotic cells and in mediating cellular entry into $\mathrm{G}_{0} /$ quiescence. Herbert Virgin (Saint Louis) showed that the autophagy molecule, Atg16 1 , is required for the exocytosis of granules containing antimicrobial peptides in Paneth cells. The corresponding abnormality seen in Atg16L1-deficient mice has also been found in Crohn's disease patients with a mutation in this molecule.

\section{Symposium B. Intrinsic Molecular Regulators of Immune-Mediated Cell Death}

Thomas Kaufmann (Bern) showed that XIAP is a crucial determinant of the switch between type II and I Fas signaling. Whereas wild-type hepatocytes were strictly dependent on Bid and mitochondrial signaling for FasL-induced apoptosis, $\mathrm{XIAP}^{-1-}$ acquired Bid-independent, type I signaling as it is known for lymphocytes. In addition, TNF-mediated fulminant hepatitis seemed to require another BH3-only protein, Bim, in addition to Bid. Solving another highly debated issue, Christoph Borner (Freiburg) showed that lysosomal

${ }^{1}$ University of Zaragoza, Zaragoza, Spain; ${ }^{2}$ University of Alberta, Alberta, Canada; ${ }^{3}$ University of Freiburg, Freiburg, Germany; ${ }^{4}$ Center of Immunology of MarseilleLuminy, Marseille, France; ${ }^{5}$ German Cancer Research Center, Heidelberg, Germany; ${ }^{6} \mathrm{~J}$ ohn Curtin School of Medical Research, Canberra, Australia; ${ }^{7}$ Aragon R + D Foundation, Aragon, Spain; ${ }^{8}$ Max-Planck Institute for Immunobiology, Freiburg, Germany and ${ }^{9}$ Peter MacCallum Cancer Center, East Melbourne, Australia ${ }^{*}$ Corresponding author: A Anel, Department Biochemistry and Molecular and Cell Biology, Faculty of Sciences, University of Zaragoza, Zaragoza 50009, Spain. Tel: +34 976 761279; Fax: + 34976 762123; E-mail: anel@ posta.unizar.es 
membrane permeability and subsequent release of cathepsins are not early events during apoptosis as proposed earlier, but depend on Bax/Bak and the apoptosome to amplify apoptosis downstream of mitochondria. Georg Häcker (Munich) unraveled that F1L, a functional $\mathrm{Bcl}-2$ homolog of the poxvirus MVA binds Bim, but instead of $\mathrm{Bim}$, the BH3-only protein, Noxa, is needed for virus-induced apoptosis in the absence of $\mathrm{F} 1 \mathrm{~L}$. He also presented some evidence that apoptosis induced by the dsRNA mimetic poly $(\mathrm{IC})$ partially required components of the intracellular MDA5/IPS1 dsRNA sensing system, thereby linking apoptosis with innate immunity. Although mitochondrial outer membrane permeabilization (MOMP) is mostly dependent on Bax and Bak, yet unknown Bax/Bak-independent mechanisms exist. Jose A Carrodeguas (Zaragoza) unveiled a new player, presenilin 1-associated protein/ mitochondrial carrier homolog 1 (PSAP/Mtch1), which seems to cause mitochondrial depolarization and apoptosis independently of Bax/Bak, likely involving the permeability transition pore. Finally, Eileen White (Piscataway) described new approaches to cancer treatment based in the combination of classic pro-apoptotic drugs and autophagy inhibitors.

\section{Symposium C. Molecular Basis and Biological Significance of Granzyme-Induced Cell Death}

This symposium moderated by Markus Simon (Freiburg), offered perhaps some of the biggest surprises at the meeting. Denis Martinvalet (Boston) began the session with work that described the capacity of intracellularly delivered granzyme A to enter mitochondria and cleave NDUFS3 (a component of the electron transport chain), leading to production of reactive oxygen species and ultimately to cell death. Christopher Froelich (Evanston) then reported that granzyme A synergized with perforin to induce rapid necrotic cell death in vitro questioning whether granzyme A was indeed cytotoxic. He then reported that granzyme A may mainly have non-cytotoxic biological properties by inducing pro-inflammatory cytokines through a caspase-1-dependent process. Thereafter, Julián Pardo (Zaragoza) extended this revitalized concept to mice, showing that isolated granzyme $\mathrm{A}$ as well as ex vivo CTL selectively expressing the granzyme could both induce pro-inflammatory cytokines. Felipe Andrade (Baltimore) then reported another non-cytotoxic effect of granzymes by showing that granzyme $\mathrm{H}$ destroys the function of critical adenoviral proteins required for viral DNA replication and granzyme $B$ inhibition. In brief presentations, Desirée Anthony (East Melbourne) reported that granzyme $M$, perhaps similar to granzyme A, modulates pro-inflammatory cytokines; Praxedis Martin (Freiburg) showed that cytotoxic T (Tc) cell-associated granzyme B, through caspase-3/-7, fragments the cytoskeletal protein, gelsolin; and Phillip Bird (Melbourne) ended the session describing the auto-regulation and extended substrate specificity of granzyme C.

In the second plenary talk, Geneviève de Saint Basile (Paris) summarized the current knowledge in the formation and secretion of cytotoxic granules in immune cells and their relationship with hemophagocytic syndromes. The molecular defects in these syndromes have allowed the identification of crucial regulators of the degranulation process: Griscelli, Chediak-Higashi, Hermansky-Pudlak 2 and familial hemophagocytic lymphohistiocytosis 3 syndrome patients have defects, respectively, in Rab27a, Lyst, AP-3 and hMunc 13-4. She described the novel two-step maturation mechanism of granules, in which perforin and granzyme B-expressing cytotoxic granules fuse with Rab27a ${ }^{+}$Rab11 ${ }^{+}$hMunc13-4 ${ }^{+}$exocytosis vesicles only in the proximity of the immunological synapse.

\section{Symposium D. Cell Death in the Development and Control of Immunity}

Rüdiger Arnold (Heidelberg) discussed one way in which CD95-independent AICD sensitivity and resistance of ATC is regulated by the kinase HPK1 (work done by D Brenner et al.). Apoptosis sensitivity of ATC is established, at least in part, by the C-terminal cleavage product of HPK1, HPK1-C, which is generated by a non-apoptotic caspase 3 activity. HPK1-C interacts with the cytoplasmic IKK complex, prevents NF- $\kappa \mathrm{B}$ translocation to the nucleus and its anti-apoptotic activity. Henning Walczak (London) described new cancer treatments based on the combination of proteasome inhibitors such as bortezomib and the death ligand, Apo2L/TRAIL. He described that bortezomib increases the sensitivity of tumors to Apo2L/TRAIL by enhancing FADD recruitment to their receptors, resulting in an increased caspase activation. In addition, he described that the incidence of DMBA/TPA-induced benign papilloma or their progression to malignant squamous cell carcinoma was not affected in mice deficient of the only apoptosisinducing murine, TRAIL-R, but that the metastasis to lymph nodes was increased, which identifies trail-r as a metastasissuppressor gene. Karsten Gülow (Heidelberg) discussed a new caspase-independent cell death pathway by inhibition of constitutively activated NF- $\kappa \mathrm{B}$ that induces ROS- and iron-dependent cell death in cutaneous $T$ cell lymphoma (CTCL; work done by $\mathrm{M}$ Kiessling et al.). Antioxidants such as $\mathrm{N}$-acetyl cysteine and glutathione or the iron chelator desferrioxamine effectively block cell death in CTCL cell lines or primary $\mathrm{T}$ cells from Sézary patients. Inhibition of constitutively active $\mathrm{NF}-\kappa \mathrm{B}$ caused downregulation of ferritin heavy chain (FHC) that led to an increase of free intracellular iron, which in turn induced massive generation of ROS through the Fenton reaction. In addition, in a murine $\mathrm{T}$ cell lymphoma model, it was shown that the inhibition of $\mathrm{NF}-\kappa \mathrm{B}$ and subsequent downregulation of $\mathrm{FHC}$ significantly delays tumor growth in vivo. These results promote $\mathrm{FHC}$ as a potential target for effective therapy in lymphomas with aberrant NF- $\kappa \mathrm{B}$ signaling.

\section{Symposium E. Cell Death as a Consequence of the Immune Response}

This session, moderated by Julián Pardo, covered not only the regulation and execution related to the effector Tc and NK cells, but also offered new views about how dead immune cells still are able to control microbes. In summarizing his group's work, Matt Mescher (Minneapolis) showed that naïve 
CD8 T cells not only need TCR and CD28 stimulation, but also a third signal provided by cytokines to get full effector functions. The next three speakers provided evidence on novel molecules involved in NK or Tc cell degranulation. Hans-Gustaf Ljunggren (Stockholm) showed that syntaxin-11 regulates NK cell degranulation. Syntaxin-11 defect resembles perforin deficiency and may explain familial hemophagocytic lymphohistiocytosis syndrome caused by syntaxin-11 deficiency. Olaf Utermoehlen (Köln) added a new molecule to the fine-tuning of granule exocytosis in Tc cells. This molecule is the lipid degrading enzyme acid sphingomyelinase and it is crucial for in vivo LCM virus control by regulating the final steps of perforin/granzyme release. Arturo Zychlinsky (Berlin) showed that even dead cells contribute as effector killers to eliminate microbes like bacteria and fungi. Throughout exquisite electron and confocal microscopy experiments, he showed that after encountering a pathogen activated neutrophils die and release structures named NETs (neutrophils extracellular traps) consisting of chromatin and granular proteins that trap and kill microbes. The session ended with three short talks in which Tiziana Daniele (Cambridge) talked about the role of Rab7 during cytotoxic cell granule exocytosis, Noa Martín-Cofreces (Madrid) offered new data on the involvement of dynein/dynactin in the movement of the microtubule organizing center in immunological synapse formation and Gideon Berke (Rehovot) presented evidence for a switch in CTL from mostly perforin mediated to largely FasL-based lytic mechanism during CTL activation in vivo.

\section{Symposium F. Tumor Immunity}

In the immunotherapy session, Tom Sayers (Frederick) described the molecular basis for synergy between the proteosome inhibitor bortezomib and TRAIL in killing the renal cancer cell line Renca and a panel of human cancer cell lines. Sensitization to botezomib/TRAIL coincided with an increased activation of caspase-8. Jan Paul Medema's presentation provided strong evidence that a defined population of stem cells can give rise to human colon cancer. As few as one human cancer stem cell (CSC) isolated from a primary tumor specimen was sufficient to generate a tumor containing multiple cell lineages when implanted into an immunosuppressed mouse. The CSC was resistant to standard chemotherapeutic agents used for human colon cancer. This resistance depended on autocrine IL-4-mediated signaling, which gave rise to an apoptosis-resistant phenotype specifically in CSC. Finally, Kees Melief presented pre-clinical and clinical data on a putative therapeutic vaccine for HPV16, one of the main causative agents of cervical and vulval cancers. A vaccine comprising overlapping peptides covering the complete sequence of the E6 and E7 oncoproteins delivered with montanide ISA 51 was able to terminate chronic infection in rabbits. Also, an ongoing trial involving 20 patients with proven HPV16 vulvar intraepithelial neoplasia had already revealed broad and sustained T cell proliferative responses with IFN $\gamma$ and IL-5 production, and a memory phenotype mimicking that seen in healthy individuals with HPV16-specific immunity. A complete clinical response accompanied by clearance of viral DNA (by PCR) was seen in 5 of the 20 patients.
The last plenary was given by Guido Kroemer, from the Gustave Roussy Institute in Villejuif. He summarized the successful efforts of his group of research to find the mechanisms by which certain anti-tumoral chemotherapies are immunogenic, and the application of the chemo-immune therapy as a more effective anti-cancer treatment. $\mathrm{He}$ described that calreticulin exposure on the surface of apoptotic cells was necessary for immunogenicity. Late release of high mobility group box 1 by apoptotic cells favors antigen processing through activation of Toll-like receptor 4 in dendritic cells.

\section{Symposium G. Cell Death Relevance During the Immune Response to Pathogens}

Matthias Regner (Canberra) reported work made in collaboration with Julián Pardo on the role of granzyme B in the Tc cell attack against target cells infected with the natural mouse pathogen, ectromelia virus, which encodes numerous inhibitors of apoptosis. However, it was shown that neither an attenuated nor a virulent strain of ectromelia virus was able to inhibit target cell apoptosis induced by Tc cells expressing granzyme B. However, when the apoptotic mitochondrial pathway was blocked, ectromelia virus inhibited granzyme B-mediated target cell apoptosis, suggesting that the pleiotropic nature of granzyme B-induced apoptosis pathways may have evolved in response to pathogen immune evasion strategies. He also reported data from in vivo cytotoxicity assays, showing that neither granzyme A nor $B$ is required for rapid and efficient in vivo target cell elimination by Tc or NK cells. Chris Bleackley (Edmonton) reported on LL-37, a human cationic antimicrobial peptide, which induces caspase-independent death in some eukaryotic cells. Both $\mathrm{Bcl}-2$ overexpressing cells, and cells deficient in Bax and Bak, were insensitive. AIF transferred to the nucleus and AIF knockdown resulted in LL-37-resistance. Lastly, chelation or inhibition of $\mathrm{Ca}^{2+}$ or calpains, respectively, inhibited killing. Together, these data show that LL-37-induced death is mediated through mitochondria in a caspase-independent, calpainand AIF-dependent manner that involves Bax activation. Granzyme-positive CTL was very sensitive to LL-37, suggesting an interesting cross talk between inflammatory and immune responses. Raymond Welsh (Worcester) reported (with M Brehm) that T cell-produced TNF restricts the initial development of virus-specific memory CD8 T cells and that type 1 interferon induces a Bim-dependent apoptosis of preexisting memory CD8 T cells at early stages of viral infection. These restrictions on the memory pool may allow for more diverse responses to other pathogens. He also showed that CD8 $\mathrm{T}$ cells in peripheral tissues were more resistant to apoptosis than those in lymphoid tissues, perhaps allowing memory $T$ cells to linger in the periphery long after infection.

\section{Symposium H. Immune Response Misdirection/ Malfunction}

Fredéric Rieux-Laucat (Paris) reviewed the genetic defects in lymphocyte cell death, and especially those in the Fas/FasL axis, resulting in autoimmune lymphoproliferative syndromes 
(ALPS). The possibility of additional undescribed elements to explain these diseases was discussed, as a fraction of patients' relatives with heterozygotic mutations in the Fas death domain (ALPS-la patients) does not show clinical manifestations. AICD compensation especially observed in patients' T cells is the consequence of perforin-mediated cell death. He also described unpublished work that casts doubts on TRAIL being one of these additional elements. Klaus Michael Debatin (UIm) described that membrane-bound FasL expressed on antigen-presenting cells prevented the expansion of $\mathrm{CD}^{+}$and $\mathrm{CD}^{+} \mathrm{T}$ cells. This was due to inhibition of proliferation on both naïve and ATC, with apoptosis induction playing a role only in the latter. The effect on naïve $T$ cells is due to inhibition of signaling events proximal to TCR ligation. These data, together with work presented by Alberto Anel (Zaragoza) in symposium $D$ on the role of FasL and Apo2L/TRAIL in the regulation of cell cycle in human $T$ cell blasts, show that death ligands can also control $\mathrm{T}$ cell proliferation, paradoxically in the absence of cell death. Ilia Voskoboinik (East Melbourne) showed that the frequent $\mathrm{A} 91 \mathrm{~V}$ or other polymorphisms in the perforin gene generate unstable forms of the protein, which are associated with late forms of $\mathrm{FHL}$ and/or hematological malignancies. Katherine Baran, from the same group, nicely complemented this talk by describing the recently discovered molecular details governing perforin polymerization.

In the last session, it was decided that the next International Cytotoxicity Workshop will be organized by Chris J Froelich (Evanston) in Chicago in 2011. 